\title{
Phenotypic Modulation of Mesenteric Vascular Smooth Muscle Cells from Type 2 Diabetic Rats is Associated with Decreased Caveolin-1 Expression
}

\author{
Maria Alicia Carrillo-Sepulveda ${ }^{\text {Takayuki Matsumoto }}{ }^{\mathrm{b}}$ \\ aDepartment of Physiology, Georgia Regents University, Augusta, GA, USA, bepartment of Physiology \\ and Morphology, Institute of Medicinal Chemistry, Hoshi University, Shinagawa-ku, Tokyo, Japan
}

\section{Key Words}

Caveolin-1 - Calponin - Goto-Kakizaki rat - PCNA - Type 2 diabetes - Mesenteric vascular smooth muscle cell

\begin{abstract}
Aims: Diabetes-induced vascular complications are associated with vascular smooth muscle cell (VSMC) phenotypic modulation, switching from a contractile to a synthetic-proliferative phenotype. Loss of caveolin-1 is involved with proliferation of VSMCs. We tested the hypothesis that mesenteric VSMCs from type 2 diabetic Goto-Kakizaki (GK) rat undergo phenotypic modulation and it is linked to decreased caveolin-1 expression. Methods: VSMCs were isolated from mesenteric arteries from GK rats and age-matched control Wistar rats. Western blotting was used to determine expression of target proteins such as caveolin-1, calponin (marker of differentiation), and proliferating cell nuclear antigen (PCNA, marker of proliferation). In addition, we measured intracellular reactive oxygen species (ROS) production using H2DCF-DA and activation of extracellular signal-regulated kinase (ERK1/2) by western blotting in VSMCs from GK stimulated with lipopolysaccharide (LPS), an endotoxin upregulated in diabetes. Results: Mesenteric VSMCs from diabetic GK rats exhibited decreased caveolin-1 and calponin expression and increased PCNA expression compared to control. Increased levels of ROS and phospho-ERK1/2 expression were also found in GK VSMCs. LPS augmented ROS and phosphorylated ERK1/2 levels to a greater extent in GK VSMCs than in control. Likewise, high glucose decreased caveolin-1 and calponin expression, increased PCNA expression and augmented ROS production in control mesenteric VSMCs. Conclusion: These results suggest that mesenteric VSMCs from diabetic GK rats undergo phenotypic modulation and it is associated with decreased caveolin-1 expression. These alterations may be due to enhanced inflammatory stimuli and glucose levels present in diabetic milieu.
\end{abstract}

Copyright (c) 2014 S. Karger AG, Basel 


\section{Introduction}

Vascular smooth muscle cells (VSMCs) from adult blood vessels display a highly specialized and differentiated phenotype. Its central function is contraction and control of vessel diameter to regulate blood flow distribution a blood pressure $[1,2]$. VSMCs in native contractile phenotype express markers of differentiation such as calponin, one of the essential proteins of the contractile apparatus in VSMCs [2, 3]. VSMCs exhibit remarkable plasticity switching from a contractile to a proliferative phenotype characterized by decreased expression of contractile proteins and increased proliferation $[2,4]$. In the vascular environment, mechanical and chemical changes lead to a rapid and reversible phenotypic modulation of VSMCs in order to maintain vascular homeostasis. It is well-established that phenotypic modulation of VSMCs is involved in the pathogenesis of atherosclerosis, one of the complications found in type 2 diabetes mellitus (T2DM) [5].

Emerging findings have revealed that patients with T2DM exhibit elevated circulatory levels of LPS [6]. LPS is an endotoxin known to stimulate reactive oxygen species (ROS) production in vascular cells [7], which is involved in the phenotypic modulation of VSMCs [8].

T2DM often is associated with obesity and hypertension and exhibits low-grade inflammatory states [9]. These complications challenge the understanding of onset of vasculopathy in T2DM. Goto-Kakizaki (GK) rats are a suitable model of nonobese T2DM [10]. We and others investigators reported that GK rats display impaired relaxation and increased contraction in various vascular beds $[11,12]$. Remodeling in resistance arteries and cerebral arteries was seen in GK rats [13-15]. Recently, Chettimada et al. [16] found that ROS lead to vascular wall remodeling and dysfunction by changing contractile protein expression in aortas from GK rats. To date, it is unknown whether or not VSMCs from mesenteric resistance arteries of GK rats display phenotypic modulation.

Caveolin, a 21-24 kDa integral membrane protein, consists of a family of at least three different isoforms: caveolin-1, -2 , and -3 , which function in the organization of caveolae and in signal-transduction $[17,18]$. Caveolin-1 has been implicated in multiple functions such as cell-cycle arrest as well as senescence, prevention of cell transformation, and anti-proliferative effects [17-19]. Changes of caveolin-1 expression are associated with pathophysiological conditions $[20,21]$. For example, decreased caveolin-1 expression was correlated with increased cell cycle entry in VSMCs from wild type mice and over-expression of caveolin-1 inhibited proliferation of VSMCs [22]. Therefore, caveolin-1 may play a role in the development of VSMCs phenotypic changes. Thus far, association between caveolin-1 and phenotypic changes in VSMCs in diabetes has not been reported.

In the present study, we hypothesized that mesenteric arterial VSMCs isolated from GK rats undergo phenotypic modulation and it is associated with changes in caveolin-1 expression and ROS levels.

\section{Materials and Methods}

\section{Animals and Experimental Procedures}

Twenty weeks-old male Wistar and GK rats were maintained on a 12-hour light and dark cycle with access to standard rodent chow and water ad libitum. The presence of diabetes was confirmed by measuring blood glucose levels using a One Touch Ultra Glucometer (Monitoring System Abbott). All experimental protocols were approved by the Institutional Animal Care and Use Committee of Georgia Regents University and conducted in accordance with the National Institutes of Health Guide for the Care and Use of Laboratory Animals. The mean plasma glucose levels in control and GK rats were $94.1 \pm 11$ and $151.5 \pm 7 \mathrm{mg} / \mathrm{dl}$, respectively. We previously reported mean arterial blood pressure to be comparable in control $(103 \pm 5 \mathrm{mmHg})$ and in GK (103 $\pm 4 \mathrm{mmHg}$ ) rats as measured by telemetry at this age [23].

Cell Cultures

VSMCs were isolated from rat mesenteric arteries by the explant method, as previously described [24]. Briefly, second and third order mesenteric arteries were cleaned of adipose and connective tissues. 
Branches of mesenteric arteries were placed in a culture dish and were maintained in Dulbecco Modified Eagle's Medium (DMEM) containing 10\% fetal bovine serum (FBS) and antibiotics in a humidified incubator at $37^{\circ} \mathrm{C}, 5 \% \mathrm{CO}_{2}$ and atmospheric $\mathrm{O}_{2}$. After 48 hours, mesenteric arteries were removed from the culture dish and cells attached to plate were maintained in cultures. VSMCs exhibited the typical "hill and valley" growth morphology and were confirmed positive (>95\%) for smooth muscle $\alpha$-actin. Cells at early passage (Passage 3) were used in all experiments. After $80 \%$ of confluence, VSMCs were serum starved for 24 hours in order to reach a quiescent state, followed by treatments with high glucose (HG, $25 \mathrm{mM}$ ) for 24, 48, and 72 hours or with normal glucose (NG, $5 \mathrm{mM}$ ) for 24 hours. To investigate the effect of HG on ROS generation, quiescent mesenteric VSMCs were treated with HG or NG for 12 hours. In another set of experiments, to measure ROS generation and ERK1/2 activation, quiescent cells were stimulated with LPS (100 ng/mL) for 15 minutes $[25,26]$.

\section{Western blotting}

Western blotting was performed to determine caveolin-1, proliferating cell number antigen (PCNA), calponin, phosho-extracellular signal-regulated kinase (ERK1/2), total ERK1/2 and $\beta$-actin protein expression. Briefly, $10 \mu \mathrm{g}$ of cellular protein was resolved by $10 \%$ sodium dodecyl sulfate polyacrylamide gel electrophoresis (SDS-PAGE), transferred to polyvinylidene difluoride (PVDF) membranes (Thermo Scientific, Rockford, IL, USA) as previously described [24] and probed with specific antibodies. Primary antibodies were follows: caveolin-1 (1:1,000) (BD Biosciences, San Jose, CA, USA); PCNA (1:1,000) (Abcam, Cambridge, UK); calponin (1:2,000) (Santa Cruz Biotechnology, Santa Cruz, CA, USA); phospho-ERK1/2 (1:1,000) (Cell Signaling Technology, Danvers, MA); total ERK1/2 (1:1,000) (Cell Signaling Technology, Danvers, MA). The stripped membranes were later probed for $\beta$-actin (1:20,000) (Sigma Aldrich, St. Louis, MO, USA) antibody as a loading control. Data are presented as fold-induction normalized to $\beta$-actin.

\section{Measurement of reactive oxygen species (ROS)}

ROS production was measured in VSMCs by 2,7-dichlorofluorescein diacetate (H2DCF-DA), as previously described [27]. Quiescent mesenteric VSMCs from GK and Wistar rats were incubated in the dark with H2DCF-DA (10 uM) for 30 minutes, followed by stimulation with LPS (100 ng/mL) for $15 \mathrm{~min}$. Additionally, quiescent mesenteric VSMCs from control rats were treated with HG or NG for 12 hours then intracellular ROS were measured. A set of ten images per each field were acquired at $20 \mathrm{x}$ magnification using a fluorescence microscopy (Axiovert 200) fitted with a camera. Then, semi-quantitative analyses were performed to detect changes in H2DCF-DA fluorescence in living VSMCs using Image Pro Plus software.

\section{Statistical Analysis}

Data are expressed as mean \pm SD. Data were analyzed with Student's $t$ test or 1 -way analysis of variance (ANOVA) followed by using Tukey post hoc test. $\mathrm{p}<0.05$ was considered statistically significant.

\section{Results}

Morphology of mesenteric VSMCs from GK and Wistar rats

When isolated in cultures, mesenteric VSMCs from control Wistar rats exhibited spindle shape (Fig. 1A) and organized arrangement at confluence (Fig.1B). Conversely, mesenteric VSMCs from GK rats lost the typical spindle shape and at confluence and displayed disarrangement (Fig. 1C and D, respectively).

Expression of caveolin-1, PCNA, and calponin in mesenteric VSMCs from GK and Wistar rats

As shown in Figs. 2A \& 2B, decreased protein expression of caveolin-1 was found in mesenteric VSMCs from the GK group compared to the Wistar group. To evaluate whether mesenteric VSMCs undergo phenotypic changes, we measured protein expression of PCNA, a marker of cell proliferation [28-30] (Figs. 2C \& 2D) and calponin (Figs. 2E \& 2F). The expression of PCNA was increased whereas the expression of calponin was decreased in GK group (vs. Wistar). 
Carrillo-Sepulveda/Matsumoto: Caveolin-1 and Phenotypic Modulation in Type 2

Diabetic Mesenteric Vascular Smooth Muscle Cells

Fig. 1. Representative photomicrographs of mesenteric VSMCs from control Wistar (A, B) and diabetic GK (C, D) rats. Top Panel: VSMCs 2 days in culture, typical spindle shape morphology. Magnification of 40x objective. Bottom panel: VSMCs 7 days in cultures. Magnification of 20x objective. Images are representative of three independent experiments.

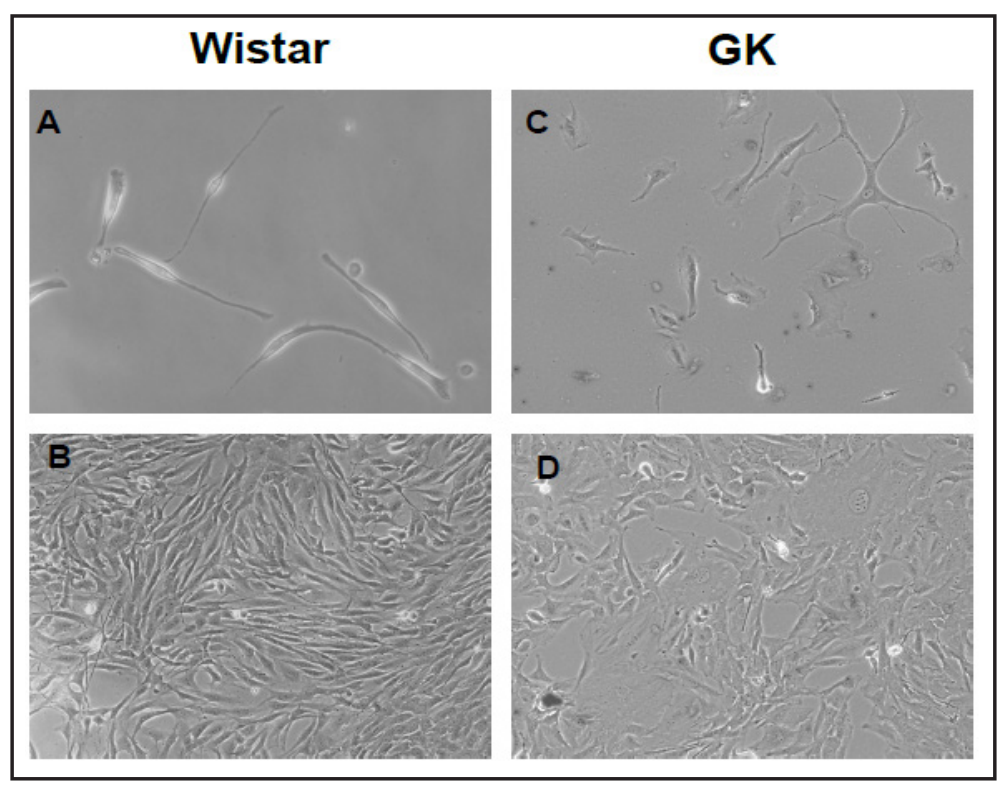

Fig. 2. Protein expression of caveolin-1, PCNA, and calponin in mesenteric VSMCs isolated from $\mathrm{GK}$ and Wistar rats. (A, C, E) Representative immunoblots showing caveolin-1 (cav-1) (A), PCNA (C), and calponin (E). (B, D, F) Intensity of bands for caveolin-1 (B), PCNA (D), and calponin (F) protein was measured by densitometric analysis and normalized by $\beta$-actin. All graphs show mean \pm SD $(n=4) .{ }^{*} \mathrm{P}<0.05$ vs. Wistar.

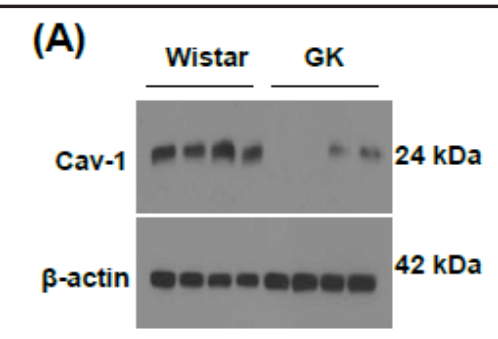

(C)

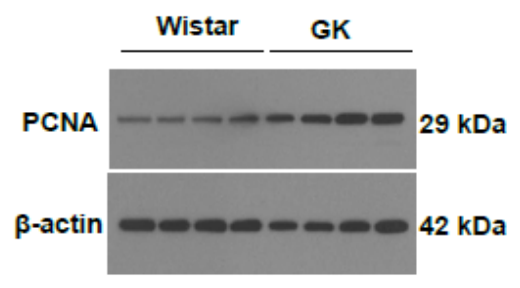

(B)

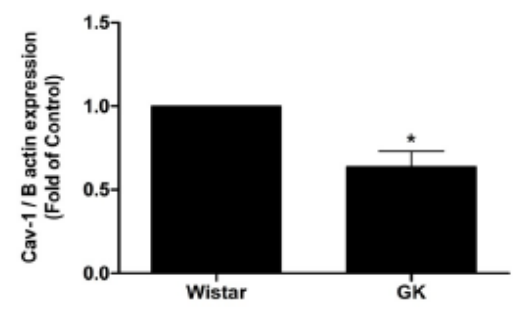

(D)

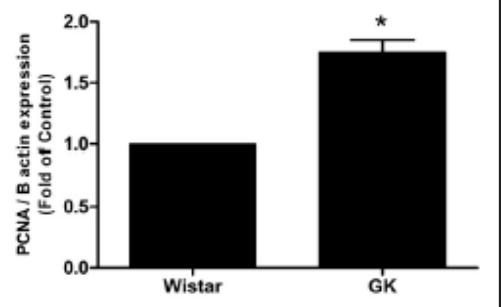

(F)

(E)

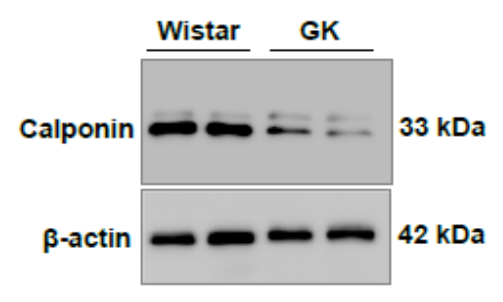

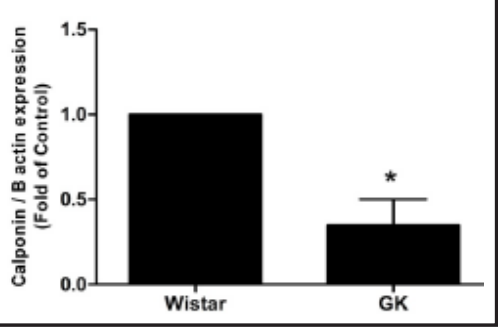

ROS production and ERK activation by inflammatory stimuli in mesenteric VSMCs from GK and Wistar rats

Since phenotypic modulation of VSMC is associated with ROS production [1] and ERK1/2 activation [3], we next examined whether LPS, which is found in elevated levels in diabetic subjects, causes alterations in ROS production and ERK1/2 activation in mesenteric 


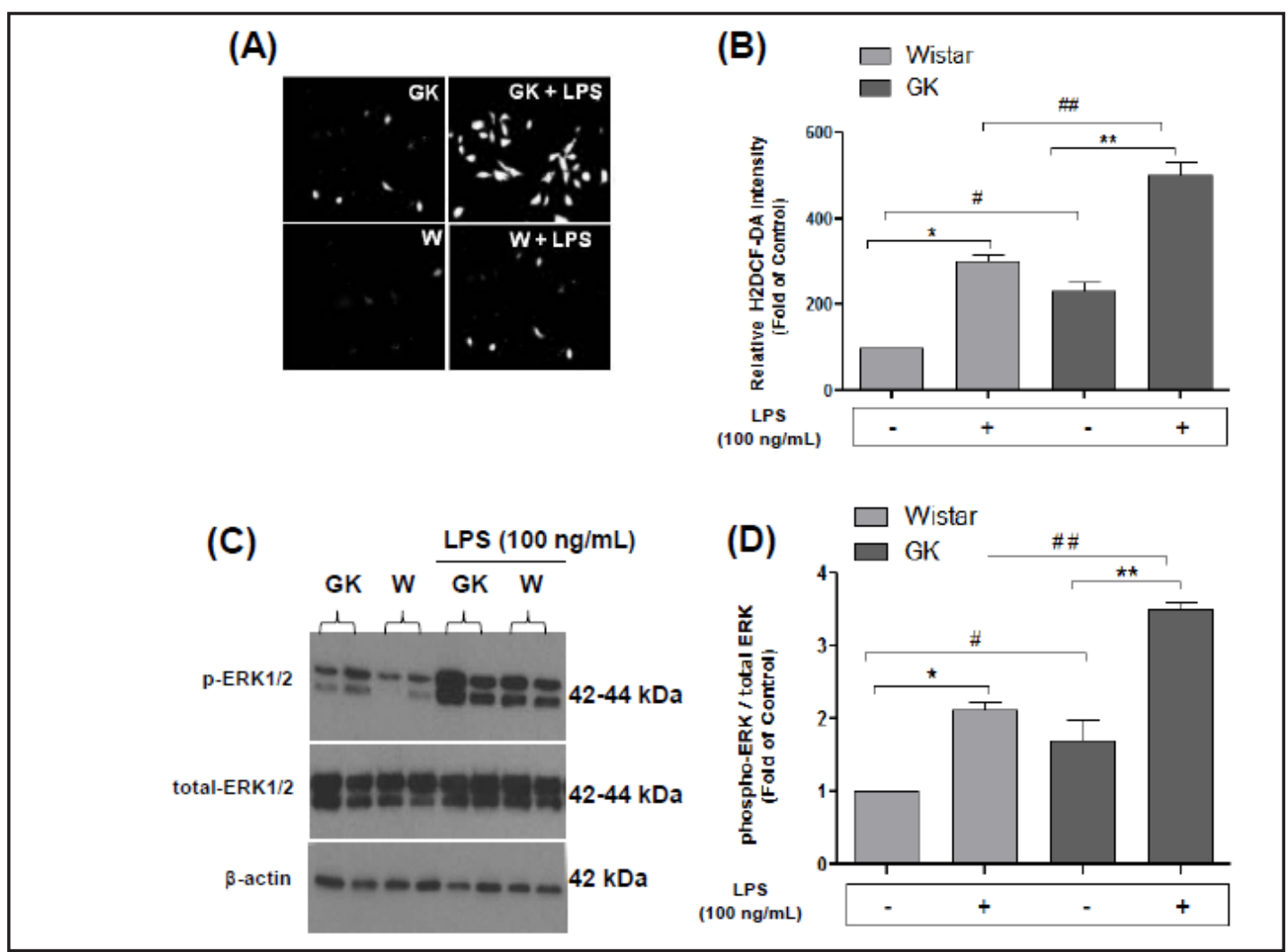

Fig. 3. LPS-induced ROS generation and ERK1/2 activation (as phosphorylated ERK1/2 measurement) in mesenteric VSMCs. Mesenteric VSMCs isolated from control Wistar and diabetic GK rats were made quiescent for 24 hours and after stimulated with or without LPS (100 ng/mL) for $15 \mathrm{~min}$ and intracellular level of ROS was measured by H2DCF-DA (A, B) and activation of ERK1/2 was measured by western blottting (C, D). (A) Photomicrographs showing changes in fluorescence (white scale) indicative of intracellular ROS generation. (B) Relative changes in fluorescence intensity were quantified and normalized by number of cells. (C) Representative immunoblots showing phosphorylated ERK1/2 (p-ERK1/2), total ERK1/2, and $\beta$-actin and intensity of bands were measured by densitometric analysis and normalized by total ERK1/2 (D). Data are expressed as mean $\pm \mathrm{SD}(n=4)$. ${ }^{*} \mathrm{P}<0.05$, $\# \mathrm{P}<0.05$ vs. un-stimulated control Wistar. ${ }^{*} \mathrm{P}<0.05$ vs. un-stimulated GK. \#\#P<0.05 vs. LPS-stimulated control cells.

VSMCs as shown in Figs. 3A and 3B. At baseline, mesenteric VSMCs from GK exhibit higher levels of ROS than mesenteric VSMCs from control rats. LPS treatment increased ROS fluorescence detection greater in mesenteric VSMCs from GK rats than in those from Wistar rats. Additionally, elevated levels of phosphorylated ERK were found in mesenteric VSMCs from GK rats and it was exacerbated after LPS treatment (Figs. 3C and D).

Effect of high glucose on caveolin-1, differentiation and proliferation in mesenteric VSMCs

High blood glucose levels are the hallmark of diabetes. In order to determine whether HG has direct effect on VSMCs phenotype and caveolin-1 expression, we evaluated caveolin-1 and phenotype-related proteins (PCNA and calponin) in mesenteric VSMCs isolated from control Wistar rats treated with HG (25 mM) for 24 (caveolin-1) and 24 to 72 (PCNA and calponin) hours. VSMCs treated with HG for 24 h significantly reduced caveolin-1 expression compared to the control (treated with $5 \mathrm{mM}$ glucose) (Figs. 4A \& B). Furthermore, HG significantly increased PCNA (Figs. 4C \& D) and decreased calponin (Figs. 4C \& E) expression in mesenteric VSMCs in a time-dependent manner. 
Carrillo-Sepulveda/Matsumoto: Caveolin-1 and Phenotypic Modulation in Type 2 Diabetic Mesenteric Vascular Smooth Muscle Cells

Fig. 4. Effect of high glucose on caveolin-1, PCNA, and calponin protein expression in mesenteric VSMCs. Quiescent mesenteric VSMCs were treated with high glucose (HG, $25 \mathrm{mM}$ ) for 24 hours (A, B) or for 24, 48, 72 hours (C, D, E) and normal glucose (NG) (control, 5 $\mathrm{mM}$ ) for 24 hours and protein expressions were measured by western blotting. (A, C) Representative immunoblots showing caveolin-1 (cav-1) (A), PCNA (C), calponin (C) and $\beta$-actin. (B, D, E) Bar graph showing relative specific bands densities of proteins to $\beta$-actin. Values (mean \pm SD, $n=4-5$ ) are expressed as fold of control NG group. ${ }^{*} \mathrm{P}<0.05$ vs. NG (control).

Fig. 5. Effect of high glucose on ROS generation in mesenteric VSMCs. Quiescent mesenteric VSMCs from control rats were treated with high glucose (HG, $25 \mathrm{mM}$ ) or normal glucose (NG) (control, $5 \mathrm{mM}$ ) for 12 hours and intracel-

\section{(B)}
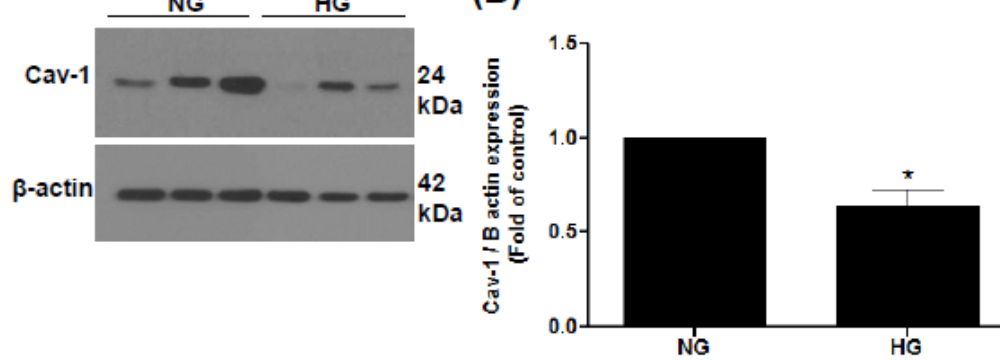

(C)

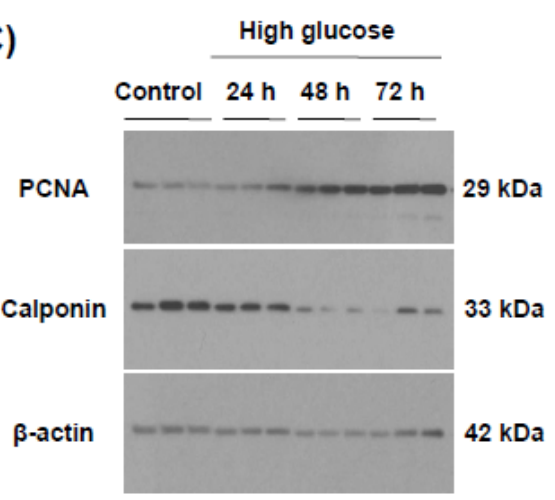

(D)

(E)
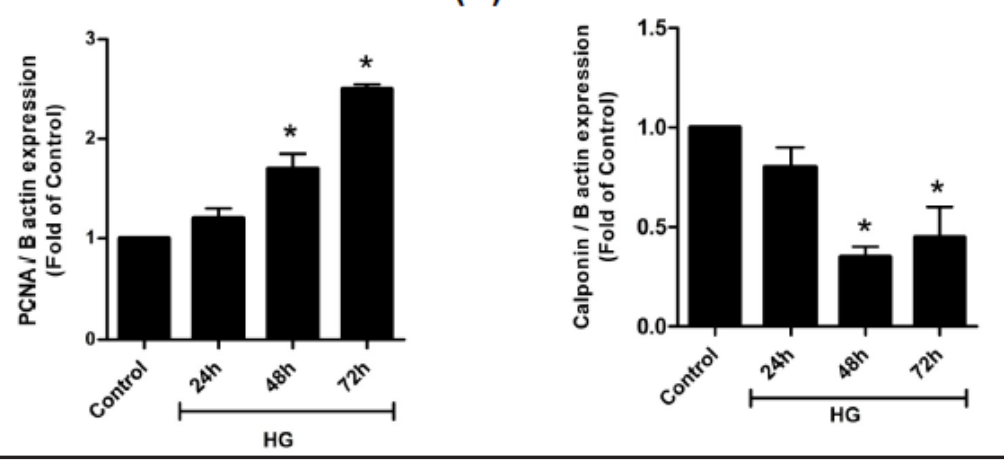

(A)

(B)
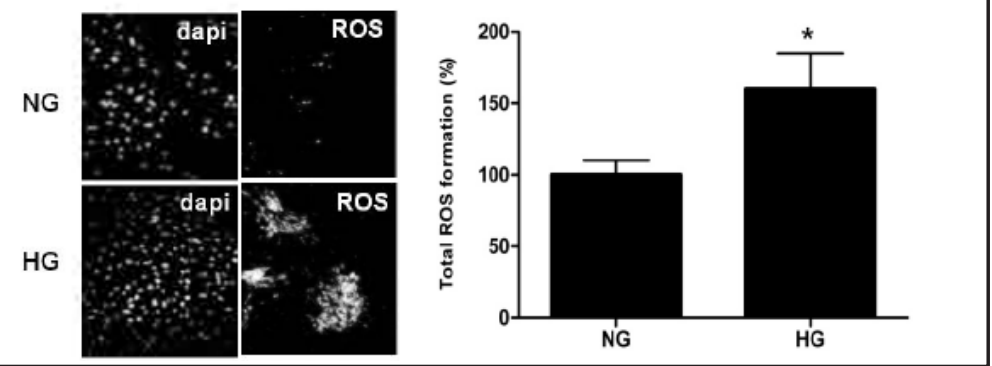

lular ROS was measured by H2DCF-DA. (A) Photomicrographs showing changes in fluorescence (white) indicative of intracelluar ROS production and relative changes in fluorescence intensity were quantified and normalized by number of cells (DAPI stain nucleus). (B) Data are shown as mean \pm SD, $n=3$. ${ }^{*} \mathrm{P}<0.05$ vs. NG.

ROS production in response to high glucose in mesenteric VSMCs

To determine whether increased ROS levels found in mesenteric VSMCs from GK may arise from hyperglycemia, we measured ROS in mesenteric VSMCs isolated from Wistar rats 
Carrillo-Sepulveda/Matsumoto: Caveolin-1 and Phenotypic Modulation in Type 2 Diabetic Mesenteric Vascular Smooth Muscle Cells

Fig. 6. Schematic summary of the present study. The imbalance of phenotype-related molecules (up-regulation of PCNA and downregulation of calponin) is seen in type 2 diabetic GK rat mesenteric vascular smooth muscle cells. This alteration results from downregulated caveolin-1 and increased ROS production and ERK1/2 activation. High glucose directly down-regulates caveolin-1 and leads to ROS production. LPS also augments ROS generation and ERK1/2 activation.

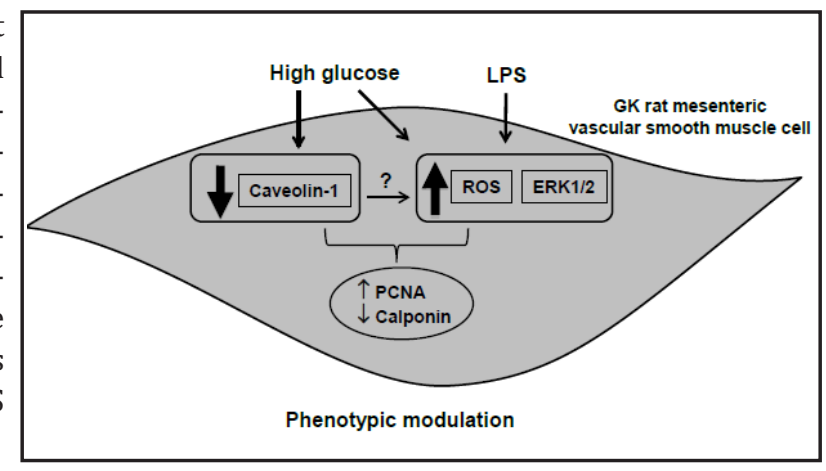

stimulated with HG for 12 hours. As shown in Fig. 5A \& 5B, production of ROS was increased by HG in mesenteric VSMCs, in accordance with previous findings in the literature $[28,31]$

\section{Discussion}

Phenotypic modulation of VSMCs plays a crucial role in vascular dysfunction in T2DM; however the mechanisms involved have not been well explored.

Although it has been difficult to mimic symptoms of diabetes, significant advances in treatment for diabetes may come from studies of suitable animal models [32,33]. A number of T2DM animal models exhibit metabolic syndrome other than DM itself including hypertension, hyperlipidemia, or obesity [32,33]. Thus, it may be difficult to clarify the relative role of each of these confounding factors in the development of DM-associated vascular dysfunction in these animal models. GK rats are a relatively unique strain as a T2DM model which does not develop obesity [10]. Studies have demonstrated that vascular dysfunction in GK rats comprise large and small arteries [11,12] and remodeling process [13-15]. However, the impact of diabetes on caveolin-1, phenotype interactions and changes in the signaling pathways in resistance arterial VSMCs in this model remain unknown.

The main finding of this study is that mesenteric VSMCs from rats with T2DM undergo phenotypic modulation and it is associated with decreased levels of caveolin-1 in parallel with enhanced levels of ROS (Fig. 6). This was supported by our results showing that GK VSMCs exhibited increased proliferation, reduced contractile protein content and augmented ROS generation.

Caveolin-1 plays pivotal roles in physiological and pathophysiological conditions [1722]. It has been demonstrated that deletion of caveolin-1 promotes VSMCs proliferation increasing intimal hyperplasia upon carotid injury [34,35]. Additionally, reduced caveolin-1 levels have been reported for plaques from hypercholesterolemic rabbits and humans [22, 36]. Lower caveolin-1 levels were also associated with features of plaque instability [37]. Therefore, caveolin-1 exerts an anti-atherosclerotic effect and may control function in VSMCs in disease conditions. In the present study, caveolin-1 and calponin were down-regulated while PCNA was upregulated in VSMCs from diabetic resistance arteries. These results suggest that the reduction of caveolin-1 expression might be closely associated with phenotypic change in GK mesenteric VSMCs.

High levels of glucose are the hallmark of diabetes. Exposure of VSMCs with HG results in increased ROS production and inflammatory agents $[28,31,38]$. In the present study, HG-treated VSMCs caused early-elevated ROS production and down-regulation of caveolin-1, which preceded alterations of phenotype-related proteins (viz. PCNA up-regulation and calponin down-regulation). Therefore, we demonstrated that HG directly leads to modulation of caveolin- 1 and phenotype-related protein expressions in mesenteric VSMCs. This conclusion is supported by the evidence showing that HG leads to a change in expression of caveolin-1 (down-regulation) in lens epithelial cells [21] and PCNA (up-regulation) in rat aortic 
VSMCs [27]. Moreover, several studies have reported that ROS and ERK1/2 are key players in the phenotypic changes observed in VSMCs [3, 5, 28, 31, 38]. ERK1/2 activation has been shown to be downstream of ROS release and previous studies demonstrated that phenotypic modulation of VSMCs is associated with ERK1/2 activation [3, 30]. Indeed, in our present study, baseline ROS production and ERK activation were increased in mesenteric VSMCs isolated from GK rats. LPS led to further ROS generation and ERK activation, which was more pronounced in GK cells. Taking our present data and other relevant evidence together, HG exposure and/or chronic inflammatory stimuli accompanied with hyperglycemia might be causal factors of caveolin-1 down-regulation and phenotypic modulation in VSMCs and these alterations were associated with ROS generation and ERK1/2 activation (Fig. 6). Although the precise mechanisms underlying the alterations of phenotype-related proteins in GK rat mesenteric VSMCs are not certain, our data suggests that these alterations might be, at least in part, due to down-regulation of caveolin-1. Future experiments will be needed to address how down-regulation of caveolin-1 play role in increased ROS levels and ERK1/2 activation.

These findings bring a new perspective to diabetic research which has been investigating alternative therapeutic targets to treat diabetic vascular complications since the actual anti-diabetic drugs do not prevent the development of vascular disorders in diabetic patients.

In summary, we found that the expression of caveolin-1 was decreased in the GK mesenteric VSMCs which might be associated with phenotypic change. These alterations of GK mesenteric VSMCs are possibly attributable to increased susceptibility to inflammatory stimuli and chronic exposure of HG levels which is characteristic of diabetes. Clarifying the signal-transduction and control of caveolin-1 expression is needed to understand the pathophysiology of diabetic vascular complications, as well as advance the treatment of T2DMassociated vascular complications.

\section{Acknowledgements}

This work was supported through a research grant from the American Heart Association to M.A.C.S (13POST14690026). The authors would like to thank R. Clinton Webb for his assistance and Adviye Ergul for her input and for provide the GK animals on this project.

\section{Disclosure Statement}

The authors have no conflict of interest.

\section{References}

1 Clempus RE, Sorescu D, Dikalova AE, Pounkova L, Jo P, Sorescu GP, Schmidt HH, Lassegue B, Griendling KK: Nox4 is required for maintenance of the differentiated vascular smooth muscle cell phenotype. Arterioscler Thromb Vasc Biol 2007;27:42-48.

-2 Owens GK, Kumar MS, Wamhoff BR: Molecular regulation of vascular smooth muscle cell differentiation in development and disease. Physiol Rev 2004;84:767-801.

-3 Carrillo-Sepulveda MA, Barreto-Chaves ML: Phenotypic modulation of cultured vascular smooth muscle cells: a functional analysis focusing on MLC and ERK1/2 phosphorylation. Mol Cell Biochem 2010;341:279289.

4 Owens GK: Regulation of differentiation of vascular smooth muscle cells. Physiol Rev 1995;75:487-517.

5 Porter KE, Riches K: The vascular smooth muscle cell: a therapeutic target in Type 2 diabetes? Clin Sci (Lond) 2013;125:167-182.

6 Jayashree B, Bibin YS, Prabhu D, Shanthirani CS, Gokulakrishnan K, Lakshmi BS, Mohan V, Balasubramanyam M: Increased circulatory levels of lipopolysaccharide (LPS) and zonulin signify novel biomarkers of proinflammation in patients with type 2 diabetes. Mol Cell Biochem 2014;388:203-210. 
7 Park HS, Chun JN, Jung HY, Choi C, Bae YS: Role of NADPH oxidase 4 in lipopolysaccharide-induced proinflammatory responses by human aortic endothelial cells. Cardiovasc Res 2006;72:447-455.

-8 Branchetti E, Poggio P, Sainger R, Shang E, Grau JB, Jackson BM, Lai EK, Parmacek MS, Gorman RC, Gorman JH, Bavaria JE, Ferrari G: Oxidative stress modulates vascular smooth muscle cell phenotype via CTGF in thoracic aortic aneurysm. Cardiovasc Res 2013;100:316-324.

-9 Lastra G, Syed S, Kurukulasuriya LR, Manrique C, Sowers JR: Type 2 diabetes mellitus and hypertension: an update. Endocrinol Metab Clin North Am 2014;43:103-122.

10 Goto Y, Kakizaki M, Masaki N: Production of spontaneous diabetic rats by repetition of selective breeding. Tohoku J Exp Med 1976;119:85-90.

11 Gupte S, Labinskyy N, Gupte R, Csiszar A, Ungvari Z, Edwards JG: Role of NAD(P)H oxidase in superoxide generation and endothelial dysfunction in Goto-Kakizaki (GK) rats as a model of nonobese NIDDM. PLoS One 2010;5:e11800.

12 Matsumoto T, Ishida K, Nakayama N, Kobayashi T, Kamata K: Involvement of NO and MEK/ERK pathway in enhancement of endothelin-1-induced mesenteric artery contraction in later-stage type 2 diabetic GotoKakizaki rat. Am J Physiol Heart Circ Physiol 2009;296:H1388-1397.

13 Sachidanandam K, Portik-Dobos V, Harris AK, Hutchinson JR, Muller E, Johnson MH, Ergul A: Evidence for vasculoprotective effects of ETB receptors in resistance artery remodeling in diabetes. Diabetes 2007;56:2753-2758.

14 Sachidanandam K, Hutchinson JR, Elgebaly MM, Mezzetti EM, Wang MH, Ergul A: Differential effects of diet-induced dyslipidemia and hyperglycemia on mesenteric resistance artery structure and function in type 2 diabetes. J Pharmacol Exp Ther 2009;328:123-130.

$\checkmark 15$ Kelly-Cobbs A, Elgebaly MM, Li W, Ergul A: Pressure-independent cerebrovascular remodelling and changes in myogenic reactivity in diabetic Goto-Kakizaki rat in response to glycaemic control. Acta Physiol (Oxf) 2011;203:245-251.

16 Chettimada S, Ata H, Rawat DK, Gulati S, Kahn AG, Edwards JG, Gupte SA: Contractile protein expression is upregulated by reactive oxygen species in aorta of Goto-Kakizaki rat. Am J Physiol Heart Circ Physiol 2014;306:H214-224.

17 Gratton JP, Bernatchez P, Sessa WC: Caveolae and caveolins in the cardiovascular system. Circ Res 2004;94:1408-1417.

18 Cohen AW, Hnasko R, Schubert W, Lisanti MP: Role of caveolae and caveolins in health and disease. Physiol Rev 2004;84:1341-1379.

19 Thyberg J: Caveolin-1 and caveolae act as regulators of mitogenic signaling in vascular smooth muscle cells. Arterioscler Thromb Vasc Biol 2003;23:1481-1483.

20 Rahman A, Sward K: The role of caveolin-1 in cardiovascular regulation. Acta Physiol (Oxf) 2009;195:231245.

21 Zhang Z, Yao K, Jin C: Apoptosis of lens epithelial cells induced by high concentration of glucose is associated with a decrease in caveolin-1 levels. Mol Vis 2009;15:2008-2017.

-22 Schwencke C, Schmeisser A, Walter C, Wachter R, Pannach S, Weck B, Braun-Dullaeus RC, Kasper M, Strasser RH: Decreased caveolin-1 in atheroma: loss of antiproliferative control of vascular smooth muscle cells in atherosclerosis. Cardiovasc Res 2005;68:128-135.

-23 Harris AK, Elgebaly MM, Li W, Sachidanandam K, Ergul A: Effect of chronic endothelin receptor antagonism on cerebrovascular function in type 2 diabetes. Am J Physiol Regul Integr Comp Physiol 2008;294:R12131219.

-24 Carrillo-Sepulveda MA, Ceravolo GS, Fortes ZB, Carvalho MH, Tostes RC, Laurindo FR, Webb RC, BarretoChaves ML: Thyroid hormone stimulates NO production via activation of the PI3K/Akt pathway in vascular myocytes. Cardiovasc Res 2010;85:560-570.

25 Chen L, Wang WY, Wang YP: Inhibitory effects of lithospermic acid on proliferation and migration of rat vascular smooth muscle cells. Acta Pharmacol Sin 2009;30:1245-1252.

-26 Lee JA, Song HY, Ju SM, Lee SJ, Kwon HJ, Eum WS, Jang SH, Choi SY, Park JS: Differential regulation of inducible nitric oxide synthase and cyclooxygenase-2 expression by superoxide dismutase in lipopolysaccharide stimulated RAW 264.7 cells. Exp Mol Med 2009;41:629-637.

27 Satoh K, Nigro P, Matoba T, O'Dell MR, Cui Z, Shi X, Mohan A, Yan C, Abe J, Illig KA, Berk BC: Cyclophilin A enhances vascular oxidative stress and the development of angiotensin II-induced aortic aneurysms. Nat Med 2009;15:649-656. 


\section{Cellular Physiology Cell Physiol Biochem 2014;34:1497-1506 and Biochemistry

Carrillo-Sepulveda/Matsumoto: Caveolin-1 and Phenotypic Modulation in Type 2 Diabetic Mesenteric Vascular Smooth Muscle Cells

28 Li H, Peng W, Zhuang J, Lu Y, Jian W, Wei Y, Li W, Xu Y: Vaspin attenuates high glucose-induced vascular smooth muscle cells proliferation and chemokinesis by inhibiting the MAPK, PI3K/Akt, and NF-kappaB signaling pathways. Atherosclerosis 2013;228:61-68.

29 Guo J, Li D, Bai S, Xu T, Zhou Z, Zhang Y: Detecting DNA synthesis of neointimal formation after catheter balloon injury in GK and in Wistar rats: using 5-ethynyl-2'-deoxyuridine. Cardiovasc Diabetol 2012;11:150.

-30 Jie W, Wang X, Zhang Y, Guo J, Kuang D, Zhu P, Wang G, Ao Q: SDF-1alpha/CXCR4 axis is involved in glucosepotentiated proliferation and chemotaxis in rat vascular smooth muscle cells. Int J Exp Pathol 2010;91:436444.

31 Ortmann J, Veit M, Zingg S, Di Santo S, Traupe T, Yang Z, Volzmann J, Dubey RK, Christen S, Baumgartner I: Estrogen receptor-alpha but not -beta or GPER inhibits high glucose-induced human VSMC proliferation: potential role of ROS and ERK. J Clin Endocrinol Metab 2011;96:220-228.

-32 Potenza MA, Nacci C, Gagliardi S, Montagnani M: Cardiovascular complications in diabetes: lessons from animal models. Curr Med Chem 2011;18:1806-1819.

33 Kumar S, Singh R, Vasudeva N, Sharma S: Acute and chronic animal models for the evaluation of anti-diabetic agents. Cardiovasc Diabetol 2012;11:9.

-34 Frank PG, Lee H, Park DS, Tandon NN, Scherer PE, Lisanti MP: Genetic ablation of caveolin-1 confers protection against atherosclerosis. Arterioscler Thromb Vasc Biol 2004;24:98-105.

-35 Hassan GS, Jasmin JF, Schubert W, Frank PG, Lisanti MP: Caveolin-1 deficiency stimulates neointima formation during vascular injury. Biochemistry 2004;43:8312-8321.

-36 Lin WW, Lin YC, Chang TY, Tsai SH, Ho HC, Chen YT, Yang VC: Caveolin-1 expression is associated with plaque formation in hypercholesterolemic rabbits. J Histochem Cytochem 2006;54:897-904.

37 Rodriguez-Feo JA, Hellings WE, Moll FL, De Vries JP, van Middelaar BJ, Algra A, Sluijter J, Velema E, van den Broek T, Sessa WC, De Kleijn DP, Pasterkamp G: Caveolin-1 influences vascular protease activity and is a potential stabilizing factor in human atherosclerotic disease. PLoS One 2008;3:e2612.

-38 Lee HS, Son SM, Kim YK, Hong KW, Kim CD: NAD(P)H oxidase participates in the signaling events in high glucose-induced proliferation of vascular smooth muscle cells. Life Sci 2003;72:2719-2730. 